\title{
Cancer Cell Targeting Using Multiple Aptamers Conjugated on Nanorods
}

\author{
Yu-Fen Huang, † Huan-Tsung Chang, ${ }^{*}$ and Weihong Tan*,† \\ Center for Research at Bio/nano Interface, Department of Chemistry and Shands Cancer Center, University of Florida, \\ Gainesville, Florida 32611, and Department of Chemistry, National Taiwan University, Taipei, Taiwan
}

Molecular recognition toward specific cells is a key issue for effective disease, such as cancer, diagnosis and therapy. Although many molecular probes such as aptamers and antibodies can recognize the unique molecular signatures of cancer cells, some of these probes only have relatively weak binding affinities. This results in poor signaling and hinders cell targeting. Here, we use $\mathrm{Au}-\mathrm{Ag}$ nanorods (NRs) as a nanoplatform for multivalent binding by multiple aptamers on the rod to increase both the signal and binding strengths of these aptamers in cancer cell recognition. Up to 80 fluorophore-labeled aptamers can be attached on a $12 \mathrm{~nm} \times 56 \mathrm{~nm} \mathrm{NR}$, resulting in a much stronger fluorescence signal than that of an individual dye-labeled aptamer probe. The molecular assembly of aptamers on the NR surfaces also significantly improves the binding affinity with cancer cells through simultaneous multivalent interactions with the cell membrane receptors. This leads to an affinity at least 26 -fold higher than the intrinsic affinity of the original aptamer probes. As determined by flow cytometric measurements, an enhancement in fluorescence signal in excess of 300fold is obtained for the NR-aptamer-labeled cells compared with those labeled by individual aptamer probes. Therefore, the molecular assembly of aptamers clearly shows potential applications for the elucidation of cells with low density of binding sites, or with relatively weak binding probes, and can thus greatly improve our ability to perform cellular imaging and targeting. This is an excellent example of using nanomaterials to develop advanced molecular binders with greatly improved properties for cellular studies.

Cancer is today's most pressing health concern. ${ }^{1}$ Cancer originates from genetic abnormalities that usually cause the affected cells to behave differently at the molecular level. Therefore, molecular probes are critical to recognize the distinct molecular features of diseased cells and to facilitate effective clinical diagnosis and treatment and mechanistic studies. Recently,

\footnotetext{
* To whom correspondence should be addressed. E-mail: $\tan @$ chem.ufl.edu. Tel and fax: (+1) 352-846-2410.

$\dagger$ University of Florida.

* National Taiwan University.

(1) Jemal, A.; Murray, T.; Ward, E.; Samuels, A.; Tiwari, R. C.; Ghafoor, A.; Feuer, E. J.; Thun, M. J. CA Cancer J. Clin. 2005, 55, 10-30.
}

a novel class of ligands, termed aptamers, has been isolated and identified for specific tumor cell recognition. Aptamers are RNA or DNA molecules that fold by intramolecular interaction into unique three-dimensional conformations for target recognition. They can be selected by a process referred to as SELEX (systematic evolution of ligands by exponential enrichment) ${ }^{2,3}$ from a pool of DNA or RNA by repetitive binding of the target molecules. Aptamers possess numerous advantageous characteristics, including small size, lack of immunogenicity, and ease of synthesis, all of which rival those of other molecular probes such as antibodies. ${ }^{4,5}$ While most aptamers reported so far have been selected for single targets, such as protein, drug, or amino acid, whole living cells have been used as targets for the selection of a panel of aptamers (cell-SELEX) for specific cell recognition. ${ }^{6-9}$ The use of cell-SELEX has resulted in a panel of aptamers for exclusive binding to the target cells, paving the way for the recognition of the unique molecular signatures of cancer cells in early diagnosis and targeted therapy.

Although a group of target cell-specific aptamers can be selected simultaneously by cell-SELEX, not all of them possess high binding affinity. Some aptamers selected from cell-SELEX have weak binding affinity and thus low signal in flow cytometry or molecular imaging, limiting their ability for sensitive detection of cancer cells. In addition, some cancer cells have a very low density of target membrane proteins for these aptamers. This is especially true during the early stages of disease development such as cancer. In this case, single-aptamer binding will not result in signal high enough to detect the minute amount of receptor sites on the cell membrane surface. Thus, in order to increase signal strength and enhance binding affinity, multivalent binding is usually considered to be the most effective approach to increase binding affinity and possibly signal strength. Scaffolds from numerous structure classes, such as proteins, dendrimers, polymers and solid supports, have been used to assemble multiple

(2) Ellington, A. D.; Szostak, J. W. Nature 1990, 346, 818-822.

(3) Tuerk, C.; Gold, L. Science 1990, 249, 505-510.

(4) Brody, E. N.; Gold, L. J. Biotechnol. 2000, 74, 5-13.

(5) Jayasena, S. D. Clin. Chem. 1999, 45, 1628-1650.

(6) Daniels, D. A.; Chen, H.; Hicke, B. J.; Swiderek, K. M.; Gold, L. Proc. Natl. Acad. Sci. U.S.A. 2003, 100, 15416-15421.

(7) Shangguan, D.; Li, Y.; Tang, Z.; Cao, Z. C.; Chen, H. W.; Mallikaratchy, P.; Sefah, K.; Yang, C. J.; Tan, W. Proc. Natl. Acad. Sci. U.S.A. 2006, 103, 11838-11843.

(8) Tang, Z. W.; Shangguan, D.; Wang, K. M.; Shi, H.; Sefah, K.; Mallikratchy, P.; Chen, H. W.; Li, Y.; Tan, W. H. Anal. Chem. 2007, 79, 4900-4907.

(9) Blank, M.; Weinschenk, T.; Priemer, M.; Schluesener, H. J. Biol. Chem. 2001, 276, 16464-16468. 
copies of recognition elements. ${ }^{10,11}$ It is desirable that the carrier of the multiple molecular probes be directly accessed to ligands with precisely controllable density and covalent conjugation.

Nanomaterials should be useful as multivalent ligand scaffolds. They provide large surface areas easily allowing the incorporation of recognition elements. In addition, they can be easily synthesized and have variable sizes. In this work, we demonstrated the use of $\mathrm{Au}-\mathrm{Ag}$ nanorods (NRs) ${ }^{12,13}$ as a platform for multiple aptamer immobilization to generate a multivalent nanoconjugate. Through covalent linkages of fluorophore-labeled aptamers on the nanorod surface, we are able to increase the binding affinity of weak-binding aptamers and also increase the overall optical signal for improved cancer cell targeting. These NR-aptamer conjugates are highly promising for use in specific cell targeting, as well as having the detection and targeting ability needed for cell studies, disease diagnosis, and therapy. In addition to all the advantages of using nanomaterial as a platform for multiple aptamer assembly, one additional reason why we choose to use NR in this work is our future plan to use aptamer conjugated NR for hyperthermia in targeted therapy as NR has excellent absorption in the IR range to generate localized heating. ${ }^{12,13}$

\section{EXPERIMENTAL SECTION}

Chemicals. Cetyltrimetylammonium bromide (CTAB), sodium tetrachloroaurate(III) dihydrate (99\%), sodium borohydride (98\%), and tris(2-carboxyethyl)phosphine (TCEP) were obtained from Sigma-Aldrich (St. Louis, MO). Ascorbic acid, glycine, Tris, and silver nitrate (99\%) were obtained from Fisher Scientific (Houston, TX). Thiol-terminated methoxypoly (ethylene glycol) (mPEG-SH, $M_{\mathrm{w}} 5000$ ) was purchased from Nanocs (New York, NY). Deoxyribonucleotides, spacer phosphoramidite 18 , and $5^{\prime}$-thiol modifiers were purchased from Glen Research (Sterling, VA). The $\mathrm{pH}$ value of the solution containing glycine $(0.5 \mathrm{M})$ was adjusted with 2.0 $\mathrm{M} \mathrm{NaOH}$ to 8.0 . Deionized water $(18.2 \mathrm{M} \Omega \cdot \mathrm{cm})$ was used to prepare all of the aqueous solutions.

Synthesis of Au NR Seeds. AuNR seeds were prepared using a slightly modified seeding method described previously. ${ }^{14} \mathrm{CTAB}$ aqueous solution $(0.2 \mathrm{M}, 5.0 \mathrm{~mL})$ was mixed with $0.5 \mathrm{mM} \mathrm{NaAuCl}$ $(5.0 \mathrm{~mL})$. Ice-cold $0.01 \mathrm{M} \mathrm{NaBH}_{4}(0.6 \mathrm{~mL})$ was added to this solution under sonication. Reaction of this mixture for $3 \mathrm{~min}$ resulted in the formation of a brownish-yellow seed solution. In the growth solution, CTAB $(0.2 \mathrm{M}, 50.0 \mathrm{~mL})$ was mixed with 1.0 $\mathrm{mM} \mathrm{NaAuCl}_{4}(50.0 \mathrm{~mL})$ and $\mathrm{AgNO}_{3}(0.1 \mathrm{M}, 0.1 \mathrm{~mL})$. After gentle mixing of the solution, $78.8 \mathrm{mM}$ ascorbic acid $(0.7 \mathrm{~mL})$ was added as a mild reducing agent. The color of the growth solution changed rapidly from dark yellow to colorless, indicating the formation of $\mathrm{AuCl}_{2}{ }^{-}$ions. Finally, a portion of the seed solution $(0.12 \mathrm{~mL})$ was added to the growth solution. The solution gradually changed color to dark pink over a period of $30 \mathrm{~min}$, indicating the formation of AuNR seeds. The as-prepared AuNR seed solutions were used directly to prepare the $\mathrm{Au}-\mathrm{Ag}$ NRs without any further purification.

(10) Kiessling, L. L.; Strong, L. E.; Gestwicki, J. E. Annu. Rep. Med. Chem. 2000, $35,321-330$.

(11) Kiessling, L. L.; Gestwicki, J. E.; Strong, L. E. Angew. Chem., Int. Ed. 2006, 45, 2348-2368

(12) Huang, Y. F.; Lin, Y. W.; Chang, H. T. Nanotechnology 2006, 17, 48854894.

(13) Huang, Y. F.; Huang, K. M.; Chang, H. T. J. Colloid Interface Sci. 2006, $301,145-154$

(14) Nikoobakht, B.; El-Sayed, M. A. Chem. Mater. 2003, 15, 1957-1962.
Synthesis of Au-Ag NRs. Aliquots $(50.0 \mathrm{~mL})$ of the asprepared AuNR seed solutions (original $\mathrm{pH} \sim 3.0$ ) were mixed with $0.2 \mathrm{M}$ glycine solutions (50.0 mL; $\mathrm{pH} 8.0$ ); note that the AuNR seed solutions still contained $\mathrm{Ag}$ and $\mathrm{Au}$ ions, as well as ascorbic acid. ${ }^{12,13}$ These mixtures were incubated without stirring at room temperature overnight to form $\mathrm{Au}-\mathrm{Ag} \mathrm{NRs}$. The sizes of the asprepared $\mathrm{CTAB}-\mathrm{Au}-\mathrm{Ag}$ NRs were verified through TEM analysis (Hitachi H7100, Tokyo, Japan); these nanocomposites appeared to be monodisperse, with average length of $56 \pm 7 \mathrm{~nm}$ and width of $12 \pm 3 \mathrm{~nm}$. A Cintra 10e double-beam UV-Vis spectrophotometer (GBC, Victoria, Australia) was used to measure the absorptions of NR solutions. The transverse and longitudinal absorption bands of the $\mathrm{CTAB}-\mathrm{Au}-\mathrm{Ag}$ NRs were centered at wavelengths of 509 and $827 \mathrm{~nm}$, respectively. The formation of $\mathrm{Au}-\mathrm{Ag}$ NRs was further confirmed by energy-dispersive X-ray, X-ray photoelectron spectroscopy, and ICPMS measurements. ${ }^{13}$

Synthesis of DNA Aptamer. The following aptamer has been selected for the CCRF-CEM: sgc8c, 5'-ATC TAA CTG CTG CGC CGC CGG GAA AAT ACT GTA CGG TTA GA-3'; K-562, KK1HO8, 5'-ATC CAG AGT GAC GCA GCA GAT CAG TCT ATC TTC TCC TGA TGG GTT CCT AGT TAT AGG TGA AGC TGG ACA CGG TGG CTT AGT-3', respectively. A high-pressure liquid chromatography (HPLC)-purified library containing a randomized sequence of 41 nucleotides was used as a control. Both of the aptamers were coupled with 5 '-thiol modifier containing 12 extra (ethylene oxide) units and labeled with fluorescein at the 3 '-end by using 3'-(6-fluorescein) CPG. An ABI3400 DNA/RNA synthesizer (Applied Biosystems, Foster City, CA) was used for the synthesis of all DNA sequences. A ProStar HPLC (Varian, Walnut Creek, CA) with a C18 column (Econosil, $5 \mu \mathrm{m}, 250 \times 4.6 \mathrm{~mm}$ ) from Alltech (Deerfield, IL) was used to purify all fabricated DNA. A Cary Bio-300 UV spectrometer (Varian, Walnut Creek, CA) was used to measure absorbances to quantify the manufactured sequences. All oligonucleotides were synthesized by solid-state phosphoramidite chemistry at a $1-\mu$ mol scale. The completed sequences were then deprotected in AMA (ammonium hydroxide/ $40 \%$ aqueous methylamine $1: 1$ ) at $65{ }^{\circ} \mathrm{C}$ for $20 \mathrm{~min}$ and further purified with reversed-phase HPLC on a C-18 column.

Functionalization of $\mathbf{A u}-\mathbf{A g} \mathbf{N R s}$. Before DNA loading, the thiol functionality on the oligonucleotides was deprotected. The 5 -thiol aptamer $(0.1 \mathrm{mM})$ was deprotected by $0.1 \mathrm{mM}$ TCEP in $50 \mathrm{mM}$ Tris- $\mathrm{HCl}$ ( $\mathrm{pH}$ 7.5) buffer for $1 \mathrm{~h}$ at room temperature. The as-prepared $\mathrm{Au}-\mathrm{Ag}$ NR solutions $(100.0 \mathrm{~mL})$ were subjected to two wash-centrifugation cycles to remove excess CTAB before DNA conjugation. Centrifugation was conducted at $12000 \mathrm{rpm}$ for $10 \mathrm{~min}$, and deionized water $(100.0 \mathrm{~mL})$ was used for washing in each cycle. The final CTAB concentration of the purified NRs $(0.25 \mathrm{nM})$ was reduced to $50.0 \mu \mathrm{M}$. To stabilize and functionalize the $\mathrm{Au}-\mathrm{Ag} \mathrm{NRs}$, we first coated them with mPEG-SH. Fresh mPEG-SH stock solutions $(2.0 \mathrm{mM})$ were prepared just before use, and $100.0 \mu \mathrm{L}$ was added to the nanorods suspension $(1.0 \mathrm{~mL})$. The mixture sat for $1 \mathrm{~h}$ at room temperature, followed by addition of $10.0 \mu \mathrm{L}$ of fluorophore-labeled alkanethiol aptamer $(0-5.0 \mu \mathrm{M})$. The mixtures were then incubated for $12 \mathrm{~h}$ and purified by centrifugation at $12000 \mathrm{rpm}$ for $5 \mathrm{~min}$.

Characterization of Fluorophore-Labeled Aptamer Binding. The loading of DNA on the NR surface was determined by fluorescence measurement (Tecan Safire microplate reader, AG) 
of fluorescein-labeled oligonucleotides. The fluorescence maximums (measured at $520 \mathrm{~nm}$ ) of the supernatant, containing free aptamers removed from the particle, were converted to molar concentrations of the fluorescein-alkanethiol modified oligonucleotide by interpolation from a standard linear calibration curve. Standard curves were prepared with known concentrations of fluorescein-labeled oligonucleotides using identical buffer $\mathrm{pH}$ and salt concentrations. Finally, the average number of oligonucleotides per particle was obtained by dividing the measured oligonucleotide molar concentration by the original NR concentration.

Cell Lines and Buffers. CCRF-CEM cells (CCL-119 T-cell, human acute lymphoblastic leukemia), Ramos cells (CRL-1596, B-cell, human Burkitt's lymphoma), and K-562 cells (CCL-243, human chronic myelogenous leukemia) were obtained from the American Type Culture Association. The cells were cultured in RPMI medium supplemented with $10 \%$ fetal bovine serum and $100 \mathrm{IU} / \mathrm{mL}$ penicillin-streptomycin. The cell density was determined using a hemocytometer, and this was performed prior to any experiments. After this, $\sim 1$ million cells dispersed in buffer [ $4.5 \mathrm{~g} / \mathrm{L}$ glucose and $5 \mathrm{mM} \mathrm{MgCl}_{2}$ in Dulbecco's PBS with calcium chloride and magnesium chloride (Sigma)] were centrifuged at $970 \mathrm{rpm}$ for $3 \mathrm{~min}$ and redispersed in the same buffer for incubation. During all experiments, the cells were kept in an ice bath at $4{ }^{\circ} \mathrm{C}$.

Flow Cytometric Analysis. To demonstrate the targeting capabilities of NR-conjugated aptamers toward specific cells, fluorescence measurements were made using a FACScan cytometer (Becton Dickinson Immunocytometry Systems, San Jose, $\mathrm{CA})$. The binding was performed by the following procedure. Approximately $1 \times 10^{6}$ of each cell type was obtained in individual test tubes. To the cell samples, $100 \mu \mathrm{L}$ of the NR solution was added, and the mixture was incubated on ice for $20 \mathrm{~min}$. After incubation, the cells were washed twice by centrifugation with $0.6 \mathrm{~mL}$ buffer and resuspended in $0.4-\mathrm{mL}$ volume of buffer. The fluorescence was determined by counting 10000 events. The fluorescein-labeled unselected ssDNA library conjugated with NRs was used as a negative control.

The binding affinity of free and NR-conjugated aptamer probes was determined by incubating CCRF-CEM cells $\left(1 \times 10^{6}\right)$ on ice for $20 \mathrm{~min}$ in the dark with varying concentrations of aptamer probes in a $200-\mu \mathrm{L}$ volume of buffer. Cells were then washed twice with $0.6 \mathrm{~mL}$ buffer, suspended in $0.4-\mathrm{mL}$ volume of buffer, and subjected to flow cytometric analysis within $30 \mathrm{~min}$. The fluoresceinlabeled unselected ssDNA library was used as a negative control to determine nonspecific binding. All of the experiments for the binding assay were repeated two to four times. The mean fluorescence intensity of target cells labeled by aptamers was used to calculate for specific binding by subtracting the mean fluorescence intensity of nonspecific binding from unselected library DNAs. ${ }^{15,16}$ The equilibrium dissociation constants $\left(K_{\mathrm{d}}\right)$ of the aptamer-cell interaction were obtained by fitting the dependence of fluorescence intensity of specific binding on the concentration of the aptamers to the equation $Y=B_{\max } X /\left(K_{\mathrm{d}}+X\right)$, using SigmaPlot (Jandel, San Rafael, CA).

(15) Davis, K. A.; Abrams, B.; Lin, Y.; Jayasena, S. D. Nucleic Acids Res. 1996, 24, 702-706.

(16) Davis, K. A.; Lin, Y.; Abrams, B.; Jayasena, S. D. Nucleic Acids Res. 1998, 26, 3915-3924.

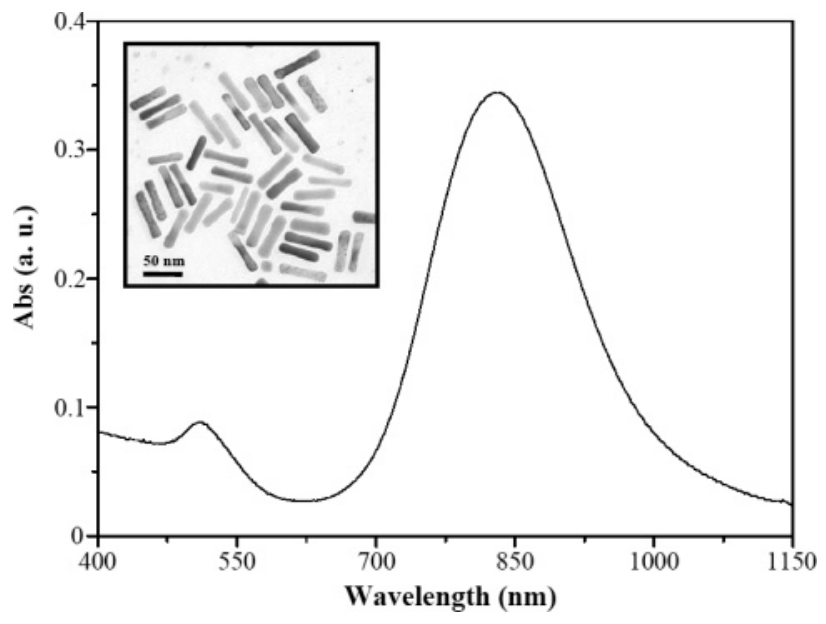

Figure 1. Absorption spectrum and TEM image of $A u-A g$ NRs. The dimensions of these nanostructures are about $12 \pm 3 \mathrm{~nm}$ in width and $56 \pm 7 \mathrm{~nm}$ in length. They possess two resonance absorption bands at 509 and $827 \mathrm{~nm}$, respectively.

Cell Imaging. For confocal imaging, the aptamers were labeled with tetramethylrhodamine anhydride (TAMRA). The treatment steps for cell incubation were the same as described in Flow Cytometric Analysis. Ten microliters of cell suspension bound with TAMRA-labeled aptamer or NR-conjugated aptamer was dropped on a thin glass slide placed above a $60 \times$ objective on the confocal microscope and then covered with a coverslip. Fluorescence imaging was conducted with a confocal microscope setup consisting of an Olympus IX-81 inverted microscope with an Olympus Fluoview 500 confocal scanning system. A 5-mW, 543-nm, He-Ne laser was the excitation source for TAMRA throughout the experiments. The objective used for imaging was a PLAPO60XO3PH $60 \times$ oil-immersion objective with a numerical aperture of 1.40 (Olympus).

\section{RESULTS AND DISCUSSION}

The NRs were fabricated with a $12 \pm 3 \mathrm{~nm}$ width and a $56 \pm$ $7 \mathrm{~nm}$ length, resulting in two resonance absorption bands at 509 and $827 \mathrm{~nm}$, respectively (Figure 1). Assembling a monolayer of PEG onto the NR surfaces can minimize aggregation and resistance to protein nonspecific binding in physiological environments. ${ }^{17,18}$ Spectrophotometric analysis revealed only a $<10 \%$ reduction in the NRs' peak absorbance after suspension in saline, confirming successful retention of nanorod stability using mPEG-SH.

Incorporation with aptamers was preformed on NR surfaces through simple thiol linkages. ${ }^{19}$ The thiol-tethered aptamer consists of three important segments: (1) the thiolalkane hydrocarbon segment, (2) the hydrophilic segment of ethylene glycol, and (3) the recognition unit of aptamer. The first segment is the hydrocarbon segment that promotes the self-assembly of a monolayer. The second segment is a hydrophilic group that separates the alkanethiol from the aptamer. The hydrophilic group is important in extending the aptamer from the surface of the

(17) Liu, Y. L.; Shipton, M. K.; Ryan, J.; Kaufman, E. D.; Franzen, S.; Feldheim, D. L. Anal. Chem. 2007, 79, 2221-2229.

(18) Liao, H.; Hafner, J. H. Chem. Mater. 2005, 17, 4636-4641.

(19) Love, J. C.; Estroff, L. A.; Kriebel, J. K.; Nuzzo, R. G.; Whitesides, G. M. Chem. Rev. 2005, 105, 1103-1170. 
monolayer with enough sufficiency to avoid steric hindrance with proteins interacting with the aptamers. ${ }^{20,21}$ Furthermore, the hydrophilic group can be designed to deter any nonspecific interactions, including the prevention of nonspecific protein interactions with monolayers supported on metal surfaces..$^{22}$ Both the lack of intermolecular repulsion between neighboring PEG moieties and the decreased interactions between the PEG and the gold surface allow high packing densities to be achieved..$^{23}$ The last segment is the molecular probe; in this case, the aptamer. Aptamer sgc8c is used for the binding to the membrane protein of CCRF-CEM cells, ${ }^{7,24}$ and other aptamers are also used for different experiments.

After exposure to different aptamer concentrations and repetitive sedimentation to wash off unbound aptamers from NRs, the conjugation efficiency of fluorescein-labeled aptamer to $\mathrm{Au}-\mathrm{Ag}$ NRs was studied (see Figure S1, Supporting Information). The fluorescence maximums (measured at $520 \mathrm{~nm}$ ) of the supernatant, containing free aptamers removed by successive washing steps, were converted to molar concentrations of the fluorescein-labeled aptamer by interpolation from a standard linear calibration curve. The result demonstrated that $\sim 80$ aptamer molecules were bound on each NR. The attachment of oligonucleotides to NRs was also confirmed by gel electrophoresis analysis. The migration velocity changes upon progressive addition of DNA. The maximum number of DNA strands loaded on each NR was determined. This correlates well with that obtained by fluorescence measurement (See Supporting Information for a more detailed description.).

By using an NR as a scaffold to attach $\sim 80$ fluorescein-labeled aptamers, the fluorescence signal of each NR-aptamer conjugate is much higher than that of individual aptamers, as displayed in Figure 2. Gold nanoparticles (AuNPs) are often considered as efficient quenchers in chromophore-AuNP composites. ${ }^{25}$ However, in our case, aptamers with 41 bases and 2 PEG linkers place a distance of $\sim 20 \mathrm{~nm}$ between the fluorophore and nanoparticle. A full coverage of NRs with well-packed PEG and fluoresceinlabeled aptamers is also helpful for stretching out the fluorophores away from the metallic surface. Therefore, the distance extends beyond the distance for efficient energy transfer, ${ }^{26}$ and the undesired fluorescence quenching in these NR-aptamer hybrids is reduced to less than $28 \%$. In addition, it has been reported that the quenching efficiency of Cy5-labeled oligonucleotides (43 bases) on gold nanoparticles is only $\sim 50 \% .{ }^{26}$ Since NRs possess lower extinction in the visible range than most nanoparticles, the quenching of the molecular excitation energy via energy transfer to the metal surface should also be considerably suppressed in these NR-aptamer conjugates.

(20) Zheng, M.; Li, Z.; Huang, X. Langmuir 2004, 20, 4226-4235.

(21) Pale-Grosdemange, C.; Simon, E. S.; Prime, K. L.; Whitesides, G. M. J. Am. Chem. Soc. 1991, 113, 12-20.

(22) Prime, K. L.; Whitesides, G. M. J. Am. Chem. Soc. 1993, 115, 1071410721.

(23) Hurst, S. J.; Lytton-Jean, A. K. R.; Mirkin, C. A. Anal. Chem. 2006, 78, 8313-8318.

(24) Shangguan, D.; Tang, Z. W.; Mallikaratchy, P.; Xiao, Z. Y.; Tan, W. H. Chembiochem 2007, 8, 603-606.

(25) Dulkeith, E.; Morteani, A. C.; Niedereichholz, T.; Klar, T. A.; Feldmann, J.; Levi, S. A.; van Veggel, F.; Reinhoudt, D. N.; Möller, M.; Gittins, D. I. Phys. Rev. Lett. 2002, 89, 2030021-2030024.

(26) Dulkeith, E.; Ringler, M.; Klar, T. A.; Feldmann, J.; MuñozJavier, A.; Parak, W. J. Nano Lett. 2005, 5, 585-589.

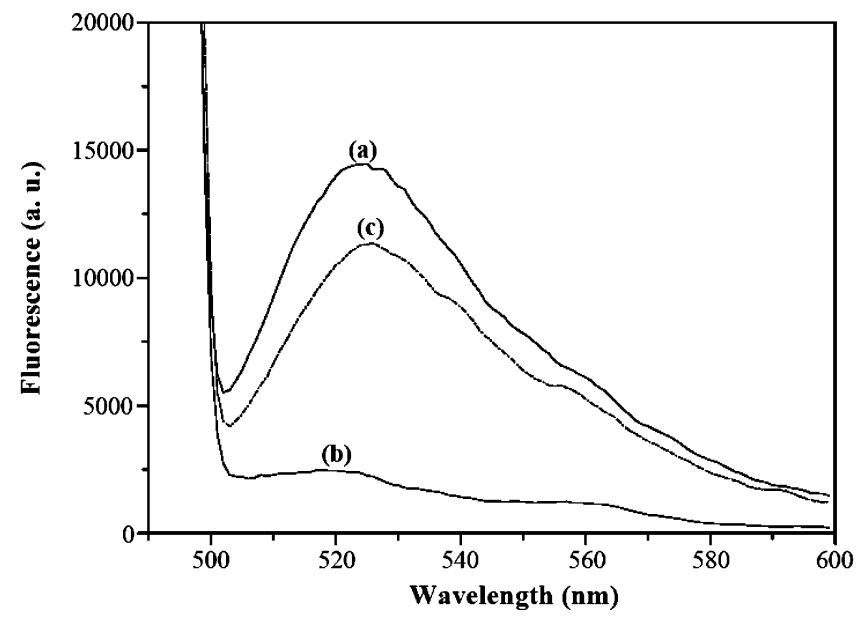

Figure 2. Fluorescence spectrum of fluorescein-labeled aptamers (solid line) at (a) 25 and (b) $0.25 \mathrm{nM}$. (c) The dashed line represents the fluorescence of NR-aptamer conjugates at $0.25 \mathrm{nM}$. Approximately 80 fluorescein-labeled aptamers were attached to each NR.

To demonstrate the specific binding of our NR-conjugated aptamers, the conjugates were added to both CEM and Ramos cell solutions, followed by flow cytometric analysis. Figure 3 shows the flow cytometric comparison of target (CCRF-CEM) cells and control negative (Ramos) cells after 20-min incubation with random DNA library (lib), sgc8c (a strong aptamer with $K_{\mathrm{d}}$ in the $\mathrm{nM}$ range), NR-lib, and NR-sgc8c. Compared to unconjugated aptamers, there is a noticeable change in the fluorescence signal observed between NR-lib and NR-sgc8c-labeled CCRFCEM cells, indicating that the binding capability of the aptamer probes is maintained well after the conjugation with NRs. No significant change in fluorescence intensity on Ramos cells was observed, further confirming the specific recognition of the $\mathrm{NR}$-aptamer conjugates for target cells.

Furthermore, we used flow cytometry to determine the binding affinity of sgc8c and NR-sgc8c to CCRF-CEM cells. As demonstrated in Figure 4, NR-sgc8c shows a higher binding affinity $\left(K_{\mathrm{d}},{ }_{\mathrm{nM}}=0.085 \pm 0.014\right)$ than that of free $\operatorname{sgc} 8 \mathrm{c}\left(K_{\mathrm{d}}, \mathrm{nM}_{\mathrm{nM}}=2.24 \pm\right.$ 0.28 ) by 26 -fold. The high binding affinity of the NR-conjugated aptamers suggests a multivalent-mediated enhancement in affinity that arises when multiple aptamers on the surface of a NR carrier bind to the receptors on the cell membrane. NR-conjugated aptamers possess a superior signal advantage over single fluorophore-labeled aptamers, which also contributes to a significant fluorescent enhancement when monitoring the binding with CCRF-CEM by confocal microscopy (see Figure S2, Supporting Information). After incubation with NR-conjugated aptamers ( 0.25 $\mathrm{nM}$ ), CEM cells presented very bright fluorescence on their periphery, whereas no fluorescence was displayed when using free TAMRA dye-labeled sgc8c in low concentration $(0.25 \mathrm{nM})$. When higher concentration of TAMRA-labeled aptamer was used, the cells could be stained for imaging as shown before. ${ }^{7}$ The $K_{d}$ for $\mathrm{Sgc} 8 \mathrm{c}$ is $\sim 2.24 \mathrm{nM}$, and a $0.25 \mathrm{nM}$ concentration of the aptamer would thus not result in a significant fluorescence for imaging of the cancer cells. It is worth noting that each nanorod has $\sim 80$ aptamers on its surface. This binding affinity incensement is significant.

Compared to individual $\mathrm{sgc} 8 \mathrm{c}, \mathrm{NR}-\mathrm{sgc} 8 \mathrm{c}$ shows at least a 33fold enhancement in fluorescence intensity with the binding of 

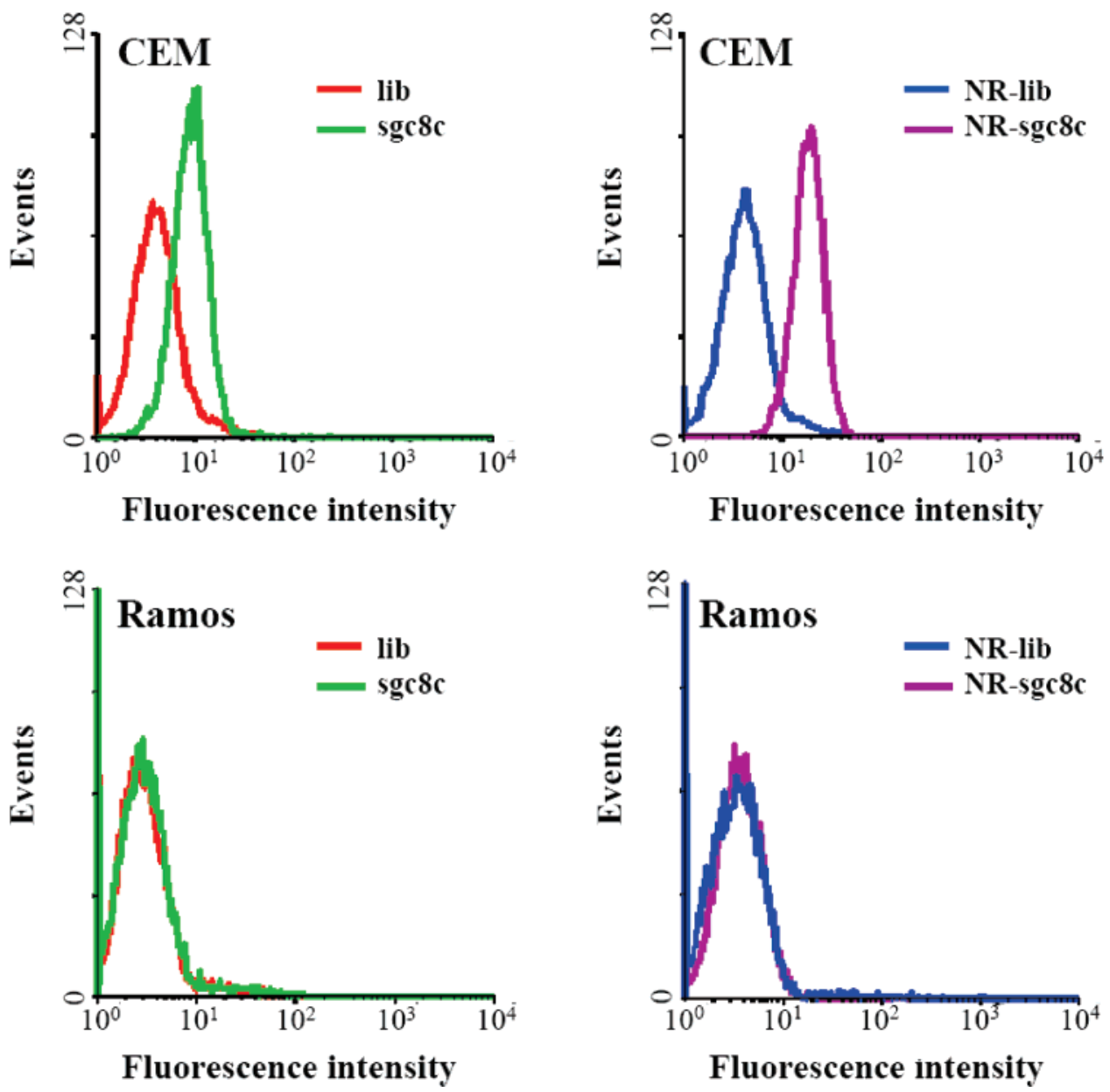

Figure 3. Flow cytometric assay to monitor the binding of sgc8c $(2.5 \mathrm{nM})$ and NR-sgc8c $(0.75 \mathrm{nM})$ with CCRF-CEM cells (target cells) and Ramos cells (control cells). The red and blue curves represent the background binding of unselected DNA library (lib) and NR-lib, respectively. For CEM cells, there was an increase in binding capacity of either unconjugated sgc8c or NR-sgc8c conjugates, whereas there was no change for the control Ramos cells (green and purple curves, respectively).

CCRF-CEM cells at low concentration range; however, no significant fluorescence difference can be observed when the signal eventually converges to the saturation limit (Figure 4). There are two possible reasons why there is little fluorescence enhancement for strong binding aptamers at high concentrations. First, multivalent interaction leads to the binding of one $\mathrm{NR}-\mathrm{sgc} 8 \mathrm{c}$ conjugate in an area with multiple target receptors on the cell membrane surface. Second, steric hindrance occurs due to the particle size, which obstructs the attachment of NR-sgc8c conjugates to each binding site on the cell membrane surface, especially when target proteins are densely located on the cell surface. In order to confirm this, we introduced free sgc8c into CCRF-CEM cells that have already been saturated with $\mathrm{NR}-\mathrm{sgc} 8 \mathrm{c}(0.75 \mathrm{nM})$. This procedure demonstrated that the fluorescent signal continues to increase as the individual sgc8c aptamer molecule is progressively added. Under these conditions, at least $50 \%$ of the binding sites are still available for further binding.

To test the feasibility of using the NR-conjugation strategy to improve the binding affinity for aptamers with weak binding capability and to increase the overall optical signal when binding with target cell, we chose one weak aptamer, KK1HO8, for testing. KK1HO8 was selected for K-562 cells and showed low binding affinity with $K_{\mathrm{d}}, \sim 296 \pm 41 \mathrm{nM}$. Figure 5 shows that only a slight shift in fluorescence intensity ( $2.87 \mathrm{au}$ ) can be observed by using

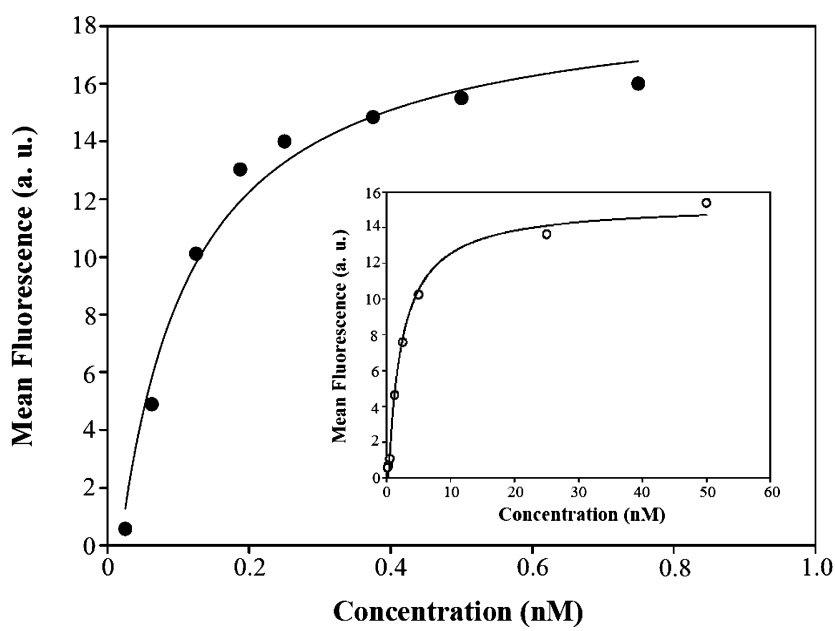

Figure 4. Flow cytometry to determine the binding affinity of NR-sgc8c (๑) and fluorescein-labeled sgc8c (O) to CCRF-CEM cells. The nonspecific binding was measured by using fluorescein-labeled unselected library DNA. The mean fluorescence intensity of target cells labeled by aptamers was obtained by subtracting the mean fluorescence intensity of nonspecific binding from unselected library DNAs.

KK1HO8 at $50 \mathrm{nM}$. However, when monitoring the binding of K-562 cells with NR-aptamer conjugates (NR-KK1HO8, 1.88 

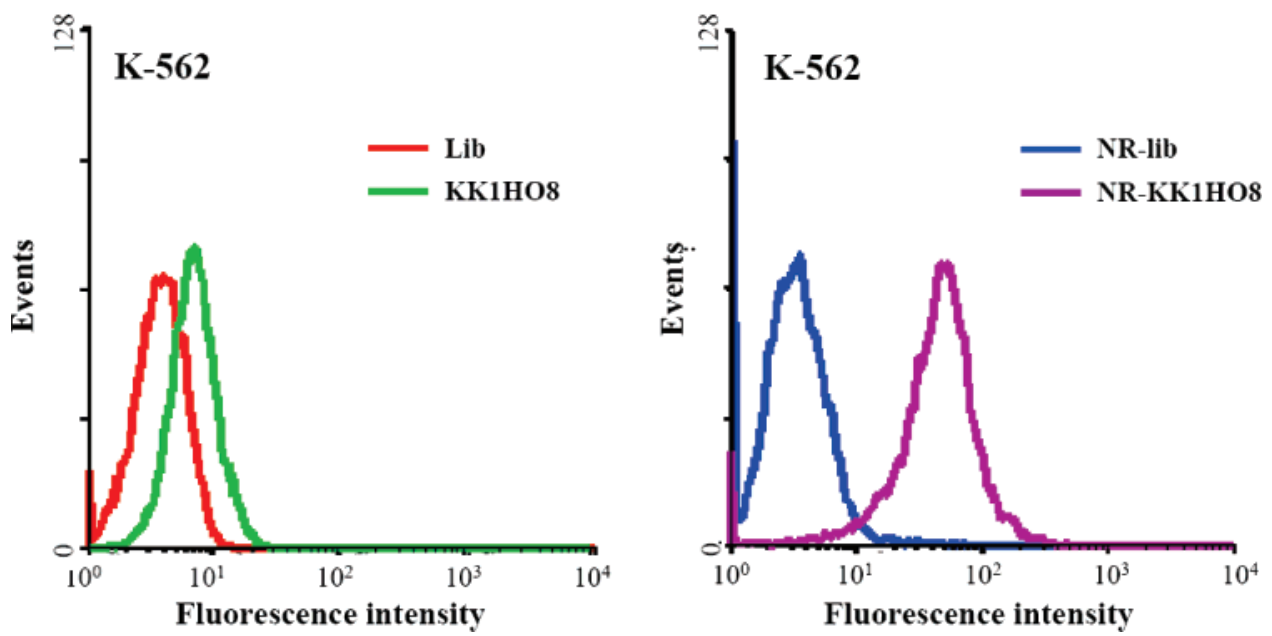

Figure 5. Binding assay of $\mathrm{KK} 1 \mathrm{HO} 8(50 \mathrm{nM})$ and $\mathrm{NR}-\mathrm{KK} 1 \mathrm{HO} 8$ conjugates ( $1.88 \mathrm{nM})$ toward $\mathrm{K}-562$ cells. The red and blue curves in the flow cytometric assay represent the background binding of unselected DNA library (lib) and NR-conjugated library (NR-lib), respectively. There was an increase in binding capacity of the NR-KK1HO8 conjugates (purple curve), whereas there was only a slight change for free KK1HO8 (green curve) with K-562 cells.

nM), significant fluorescence intensity change (39.74 au) was observed. The fluorescence change is larger than the maximum intensity (14.22 au) that could be achieved when K-562 cells were saturated with KK1HO8 (500 nM). We have calculated that there is a 300 -fold enhancement in fluorescence intensity of NR-KK1HO8-labeled cells compared to KK1HO8-labeled cells. This result clearly shows the excellent binding capability and the intense light-emitting property of the NR-aptamer conjugates.

\section{CONCLUSION}

In summary, we demonstrated the use of NR as an efficient and robust multivalent platform for molecular assembly of aptamers for target cell recognition. Simultaneous attachment of up to 80 fluorophore-labeled aptamers onto the functionalized NR surface creates an assembly of multivalent binding probes with significant fluorescence intensity enhancement for molecular recognition. The target cells can be clearly visualized through the strong fluorescence due to the multiple aptamers for molecular imaging of cells. The binding affinity of NR-aptamer conjugates has been increased by $\sim 26$-fold compared to that of single aptamer molecules. Overall, these NR-aptamer conjugates show high fluorescence signal, minimal nonspecific binding, and strong binding with target cells. The NR-aptamer conjugate has greatly enhanced binding affinity to carcinoma cells. These properties make NR-aptamer conjugates highly promising for cancer cell studies and for targeted therapy as the NR has strong IR absorption for hyperthermia in photothermal therapy.

\section{ACKNOWLEDGMENT}

This work was supported by NIH NCI and NIGMS grants, and by State of Florida Center for NanoBiosensors. Y.-F.H. acknowledges the support from the National Science Council (NSC095SAFI564625TMS) of Taiwan.

\section{SUPPORTING INFORMATION AVAILABLE}

Supplementary figures. This material is available free of charge via the Internet at http://pubs.acs.org.

Received for review November 10, 2007. Accepted December 11, 2007.

AC702322J 